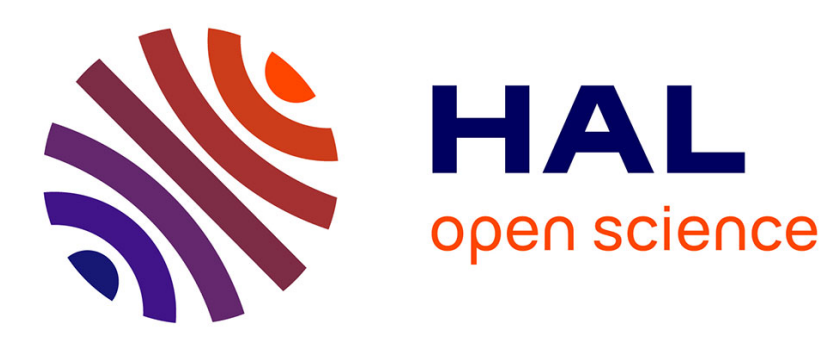

\title{
Bohr-Neugebauer type theorem for some partial neutral functional differential equations
}

\author{
Mostafa Adimy, Abdelhai Elazzouzi, Khalil Ezzinbi
}

\section{To cite this version:}

Mostafa Adimy, Abdelhai Elazzouzi, Khalil Ezzinbi. Bohr-Neugebauer type theorem for some partial neutral functional differential equations. 2006. hal-00258398

\section{HAL Id: hal-00258398 \\ https://hal.science/hal-00258398}

Preprint submitted on 22 Feb 2008

HAL is a multi-disciplinary open access archive for the deposit and dissemination of scientific research documents, whether they are published or not. The documents may come from teaching and research institutions in France or abroad, or from public or private research centers.
L'archive ouverte pluridisciplinaire HAL, est destinée au dépôt et à la diffusion de documents scientifiques de niveau recherche, publiés ou non, émanant des établissements d'enseignement et de recherche français ou étrangers, des laboratoires publics ou privés. 


\title{
Bohr-Neugebauer type theorem for some partial neutral functional differential equations *
}

\author{
Mostafa Adimy \\ Universitéde Pau et des Pays de l'Adour, Laboratoire de Mathématiques \\ Appliquées IPRA, FRE 2570, Avenue de l'université 64000 Pau, France
}

Abdelhai Elazzouzi, Khalil Ezzinbi

Université Cadi Ayyad, Facultédes Sciences Semlalia, Département de

Mathématiques, B.P. 2390, Marrakesh, Morocco

\begin{abstract}
In this work, we study the existence of almost periodic solutions for some partial neutral functional differential equations. Using the variation of constants formula and the spectral decomposition of the phase space developed in [6], we prove that the existence of an almost periodic solution is equivalent to the existence of a bounded solution on $\mathbb{R}^{+}$.
\end{abstract}

Key words:

neutral equation, Hille-Yosida condition, semigroup, variation of constants

formula, essential growth, spectral decomposition, almost periodic solution.

ऋ This research is supported by TWAS Grant under contract: No: 03-030 RG/MATHS/AF/AC

To appear in Nonlinear Analysis, Theory, Methods and Applications

Email addresses: mostafa.adimy@univ-pau.fr (Mostafa Adimy), ezzinbi@ucam.ac.ma (Khalil Ezzinbi).

Preprint submitted to Elsevier Preprint 13 March 2006 


\section{Introduction}

The purpose of this work is to study the existence of almost periodic solutions of the following class of partial neutral functional differential equations

$$
\left\{\begin{array}{l}
\frac{d}{d t} \mathcal{D} u_{t}=A \mathcal{D} u_{t}+L\left(u_{t}\right)+f(t), \text { for } t \geq \sigma, \\
u_{\sigma}=\varphi \in C:=C([-r, 0] ; X),
\end{array}\right.
$$

where $A$ is a linear operator on a Banach space $X$, not necessarily densely defined and satisfies the known Hille-Yosida condition:

$\left(\mathbf{H}_{\mathbf{0}}\right)$ there exist $\bar{M} \geq 0$ and $\omega \in \mathbb{R}$ such that $(\omega,+\infty) \subset \rho(A)$ and

$$
\left|R(\lambda, A)^{n}\right| \leq \frac{\bar{M}}{(\lambda-\omega)^{n}}, \quad n \in \mathbb{N}, \lambda>\omega,
$$

where $\rho(A)$ is the resolvent set of $A$ and $R(\lambda, A)=(\lambda I-A)^{-1}$, for $\lambda \in \rho(A)$. $C$ is the space of continuous functions from $[-r, 0]$ to $X$ endowed with the uniform norm topology. $\mathcal{D}: C \rightarrow X$ is a bounded linear operator which has the following form

$$
\mathcal{D} \varphi:=\varphi(0)-\int_{-r}^{0}[d \eta(\theta)] \varphi(\theta), \varphi \in C
$$

for a mapping $\eta:[-r, 0] \rightarrow \mathcal{L}(X)$ of bounded variation and non atomic at zero, which means that there exists a continuous nondecreasing function $\delta:[0, r] \rightarrow[0,+\infty)$ such that $\delta(0)=0$ and

$$
\left|\int_{-s}^{0}[d \eta(\theta)] \varphi(\theta)\right| \leq \delta(s) \sup _{-r \leq \theta \leq 0}|\varphi(\theta)|, \varphi \in C, s \in[0, r]
$$

where $\mathcal{L}(X)$ denotes the space of bounded linear operators from $X$ to $X$. For every $t \geq \sigma$, the history function $u_{t} \in C$ is defined by

$$
u_{t}(\theta)=u(t+\theta) \text {, for } \theta \in[-r, 0] .
$$

$L$ is a bounded linear operator from $C$ into $X$ and $f$ is a continuous function from $\mathbb{R}$ to $X$.

In [26] and [27], the authors studied a system of partial neutral functional differential-difference equations defined on the unit circle $S$, which is a model for a continuous circular array of resistively coupled transmission lines with mixed initial boundary conditions. This system is

$$
\frac{\partial}{\partial t}[u(., t)-q u(., t-r)]=k \frac{\partial^{2}}{\partial x^{2}}[u(., t)-q u(., t-r)]+\zeta\left(u_{t}\right), \text { for } t \geq 0,
$$


where $x \in S, k$ is a positive constant, $\zeta$ is a continuous function and $0 \leq q<1$. The phase space is $C\left([-r, 0] ; H^{1}(S)\right)$. In [16] and [17], the author studied the qualitative behavior of solutions of Equation (2). He obtained several results about stability, attractiveness and bifurcation of solutions near an equilibrium. In [2], motivated by the above works, the authors gave the basic theory for the following partial neutral functional differential equation

$$
\left\{\begin{array}{l}
\frac{d}{d t}[u(t)-F u(t-r)]=A[u(t)-F u(t-r)]+P\left(u_{t}\right), t \geq 0, \\
u_{0}=\varphi \in C,
\end{array}\right.
$$

where $A$ is not necessarily densely defined and satisfies the Hille-Yosida condition on a Banach space $X, F$ is a bounded linear operator from $X$ to $X$ and $P$ is a bounded linear operator from $C$ to $X$. It was proved in particular, that the solutions generate a locally Lipschitz continuous integrated semigroup. In [4] and [5], the authors studied the existence, regularity and stability of solutions for a more general class of nonlinear partial neutral functional differential equations.

The existence of periodic solutions or almost periodic solutions is very important in the qualitative studies of many problems. Among numerous results in this topics, we mention the following result which is classical in the theory of ordinary differential equations. Let us consider the following system of differential equations in finite dimensional space

$$
\frac{d}{d t} x(t)=B x(t)+g(t), t \in \mathbb{R}
$$

where $B$ is a constant $n \times n$-matrix and $g: \mathbb{R} \rightarrow \mathbb{R}^{n}$ is a continuous and $\omega$ periodic function. In [20], Massera studied the existence of periodic solutions of Equation (3). He proved the equivalence between the existence of bounded solutions on $\mathbb{R}^{+}$and the existence of $\omega$-periodic solutions. As a generalization of this result, Bohr and Neugebauer, see [13], studied the existence of almost periodic solutions of Equation (3) in the case where the function $g$ is almost periodic. More precisely, they proved that the existence of a bounded solution on $\mathbb{R}^{+}$implies the existence of an almost periodic solution and every bounded solution on $\mathbb{R}$ is almost periodic. Another direction of generalization of these two classical results is to discuss the existence of periodic or almost periodic solutions for partial functional differential equations (i.e. Equation (1) in the case $\mathcal{D}(\varphi)=\varphi(0))$. Many works are devoted to this subject. For more information, we refer to [8], [9], [12], [14], [18] and [21].

In [6], the authors discussed the fundamental linear theory of Equation (1). In particular, they studied the asymptotic behavior of the solution semigroup 
of the homogeneous equation

$$
\left\{\begin{array}{l}
\frac{d}{d t} \mathcal{D} u_{t}=A \mathcal{D} u_{t}+L\left(u_{t}\right), \text { for } t \geq 0 \\
u_{0}=\varphi \in C
\end{array}\right.
$$

They obtained a new variation of constants formula associated to Equation (1). Moreover, they established the existence of periodic and almost periodic solutions in the case where the semigroup associated to Equation (4) is hyperbolic.

The goal of this work is to prove the existence of almost periodic solutions of Equation (1) without the hyperbolicity condition. More precisely, we will show that the existence of an almost periodic solution of Equation (1) is equivalent to the existence of a bounded solution on $\mathbb{R}^{+}$. Our approach is based on the variation of constants formula and the spectral decomposition of the phase space developed in [6].

This work is organized as follows: in Section 2, we recall the variation of constants formula obtained in [6]. In section 3, we develop several fundamental results about the spectral decomposition of solutions of Equation (1). As a consequence, we obtain a finite dimensional reduction of Equation (1). In Section 4 , we prove the main result of this work which states the equivalence between the existence of bounded solutions on $\mathbb{R}^{+}$and the existence of almost periodic solutions of Equation (1). To illustrate our approach, we propose an application for the model (2).

\section{Variation of constants formula}

Throughout this paper, we suppose that the operator $A: D(A) \subset X \longrightarrow X$ satisfies the Hille-Yosida condition $\left(\mathbf{H}_{\mathbf{0}}\right)$.

We need the following definition and results which are taken from [4] and [6].

Definition 1 [6] A continuous function u from $[-r+\sigma,+\infty)$ into $X$ is an integral solution of Equation (1), if

(i) $\int_{\sigma}^{t} \mathcal{D} u_{s} d s \in D(A)$, for $t \geq \sigma$,

(ii) $\mathcal{D} u_{t}=\mathcal{D} \varphi+A \int_{\sigma}^{t} \mathcal{D} u_{s} d s+\int_{\sigma}^{t}\left[L\left(u_{s}\right)+f(s)\right] d s, \quad$ for $t \geq \sigma$,

(iii) $u_{\sigma}=\varphi$.

From the closedness property of the operator $A$, we can see that if $u$ is an integral solution of Equation (1), then $\mathcal{D} u_{t} \in \overline{D(A)}$ for all $t \geq \sigma$. In particular, $\mathcal{D} \varphi \in \overline{D(A)}$. 
Let us introduce the part $A_{0}$ of the operator $A$ in $\overline{D(A)}$ defined by

$$
\left\{\begin{array}{l}
D\left(A_{0}\right)=\{x \in D(A): A x \in \overline{D(A)}\} \\
A_{0} x=A x, \text { for } x \in D\left(A_{0}\right)
\end{array}\right.
$$

Lemma 2 [7, Lemma 3.3.12, pp. 140] Assume that $\left(\mathbf{H}_{0}\right)$ holds. Then $A_{0}$ generates a strongly continuous semigroup $\left(T_{0}(t)\right)_{t \geq 0}$ on $\overline{D(A)}$.

The integrated form of Equation (1) is given by the next result.

Theorem 3 [4] Assume that $\left(\mathbf{H}_{\mathbf{0}}\right)$ holds. Then, for all $\varphi \in C$ such that $\mathcal{D} \varphi \in \overline{D(A)}$, Equation (1) has a unique integral solution $u$ on $[-r+\sigma,+\infty)$. Moreover, $u$ is given by

$$
\mathcal{D} u_{t}=T_{0}(t-\sigma) \mathcal{D} \varphi+\lim _{\lambda \rightarrow+\infty} \int_{\sigma}^{t} T_{0}(t-s) B_{\lambda}\left[L\left(u_{s}\right)+f(s)\right] d s, \text { for } t \geq \sigma,
$$

where $B_{\lambda}=\lambda R(\lambda, A)$, for $\lambda>\omega$.

In the sequel of this work, $u(., \sigma, \varphi, f)$ denotes the integral solution of Equation (1). The phase space $C_{0}$ of Equation (1) is given by

$$
C_{0}:=\{\varphi \in C: \mathcal{D} \varphi \in \overline{D(A)}\}
$$

For each $t \geq 0$, we define the linear operator $\mathcal{U}(t)$ on $C_{0}$ by

$$
\mathcal{U}(t) \varphi=v_{t}(., \varphi)
$$

where $v(., \varphi)$ is the integral solution of the homogeneous equation (4). We have the following result.

Proposition 4 [6, Proposition 2] Assume that $\left(\mathbf{H}_{\mathbf{0}}\right)$ holds. Then $(\mathcal{U}(t))_{t \geq 0}$ is a strongly continuous semigroup on $C_{0}$, that is:

(i) for all $t \geq 0, \mathcal{U}(t)$ is a bounded linear operator on $C_{0}$,

(ii) $\mathcal{U}(0)=I$,

(iii) $\mathcal{U}(t+s)=\mathcal{U}(t) \mathcal{U}(s)$, for all $t, s \geq 0$,

(iv) for all $\varphi \in C_{0}, \mathcal{U}(t) \varphi$ is a continuous function of $t \geq 0$ with values in $C_{0}$. Moreover,

(v) $(\mathcal{U}(t))_{t \geq 0}$ satisfies, for $\varphi \in C_{0}, t \geq 0$ and $\theta \in[-r, 0]$, the following translation property

$$
(\mathcal{U}(t) \varphi)(\theta)=\left\{\begin{array}{l}
(\mathcal{U}(t+\theta) \varphi)(0) \quad \text { if } t+\theta \geq 0 \\
\varphi(t+\theta) \text { if } t+\theta \leq 0
\end{array}\right.
$$

We investigate, in the next result, the infinitesimal generator of $(\mathcal{U}(t))_{t \geq 0}$. 
Theorem 5 [6, Theorem 3] Assume that $\left(\mathbf{H}_{\mathbf{0}}\right)$ holds. Then the operator $\mathcal{A}_{\mathcal{U}}$ defined on $C_{0}$ by

$$
\left\{\begin{array}{l}
D\left(\mathcal{A}_{\mathcal{U}}\right)=\left\{\varphi \in C^{1}([-r, 0] ; X): \mathcal{D} \varphi \in D(A), \mathcal{D} \varphi^{\prime} \in \overline{D(A)} \text { and } \mathcal{D} \varphi^{\prime}=A \mathcal{D} \varphi+L(\varphi)\right\}, \\
\mathcal{A}_{\mathcal{U}} \varphi=\varphi^{\prime}, \text { for } \varphi \in D\left(\mathcal{A}_{\mathcal{U}}\right)
\end{array}\right.
$$

is the infinitesimal generator of the semigroup $(\mathcal{U}(t))_{t \geq 0}$ on $C_{0}$.

In order to give a variation of constants formula associated to Equation (1), we need to extend the semigroup $(\mathcal{U}(t))_{t \geq 0}$ to the space $C_{0} \oplus\left\langle X_{0}\right\rangle$ where $\left\langle X_{0}\right\rangle$ is the space defined by

$$
\left\langle X_{0}\right\rangle=\left\{X_{0} c: c \in X\right\},
$$

the function $X_{0} c$ is given, for $c \in X$, by

$$
\left(X_{0} c\right)(\theta)= \begin{cases}0 \text { if } & \theta \in[-r, 0) \\ c \text { if } & \theta=0\end{cases}
$$

The space $C_{0} \oplus\left\langle X_{0}\right\rangle$ equipped with the norm $\left\|\phi+X_{0} c\right\|_{\widetilde{A}_{u}}=|\phi|+|c|$, for $(\phi, c) \in C_{0} \times X$, is a Banach space. Consider the extension $\widetilde{\mathcal{A}}_{\mathcal{U}}$ of the operator $\mathcal{A}_{\mathcal{U}}$ on $C_{0} \oplus\left\langle X_{0}\right\rangle$ defined by

$$
\left\{\begin{array}{l}
D\left(\widetilde{\mathcal{A}}_{\mathcal{U}}\right)=\left\{\varphi \in C^{1}([-r, 0] ; X): \mathcal{D} \varphi \in D(A) \text { and } \mathcal{D} \varphi^{\prime} \in \overline{D(A)}\right\} \\
\widetilde{\mathcal{A}}_{\mathcal{U}} \phi=\varphi^{\prime}+X_{0}\left(A \mathcal{D} \varphi+L \varphi-\mathcal{D} \varphi^{\prime}\right)
\end{array}\right.
$$

In order to compute the resolvent operator $R\left(\lambda, \widetilde{\mathcal{A}}_{\mathcal{U}}\right)$, we need to make the following assumption

$\left(\mathbf{H}_{\mathbf{1}}\right) \mathcal{D}\left(e^{\lambda \cdot} c\right) \in D(A)$, for all $c \in D(A)$ and all complex $\lambda$, where $e^{\lambda \cdot} c \in C$ is defined by

$$
\left(e^{\lambda \cdot} c\right)(\theta)=e^{\lambda \theta} c \text {, for } \theta \in[-r, 0] .
$$

Lemma 6 [6, Theorem 13] Assume that $\left(\mathbf{H}_{0}\right)$ and $\left(\mathbf{H}_{1}\right)$ hold. Then $\widetilde{\mathcal{A}}_{\mathcal{U}}$ satisfies the Hille-Yosida condition on $C_{0} \oplus\left\langle X_{0}\right\rangle$ : there exist $\widetilde{M} \geq 0$ and $\widetilde{\omega} \in \mathbb{R}$ such that $(\widetilde{\omega},+\infty) \subset \rho\left(\widetilde{\mathcal{A}}_{\mathcal{U}}\right)$ and

$$
\left|R\left(\lambda, \widetilde{\mathcal{A}}_{\mathcal{U}}\right)^{n}\right| \leq \frac{\widetilde{M}}{(\lambda-\widetilde{\omega})^{n}}, \quad n \in \mathbb{N}, \lambda>\widetilde{\omega} .
$$

Now, we can state the variation of constants formula associated to Equation (1). 
Theorem $\mathbf{7}$ [6, Theorem 16] Assume that $\left(\mathbf{H}_{\mathbf{0}}\right)$ and $\left(\mathbf{H}_{\mathbf{1}}\right)$ hold. Then, for all $\varphi \in C_{0}$, the integral solution $u$ of Equation (1) is given by the following variation of constants formula

$$
u_{t}=\mathcal{U}(t-\sigma) \varphi+\lim _{\lambda \rightarrow+\infty} \int_{\sigma}^{t} \mathcal{U}(t-s)\left(\widetilde{B}_{\lambda}\left(X_{0} f(s)\right)\right) d s, \text { for } t \geq \sigma,
$$

where $\widetilde{B}_{\lambda}=\lambda R\left(\lambda, \widetilde{\mathcal{A}}_{\mathcal{U}}\right)$ for $\lambda>\widetilde{\omega}$.

\section{Spectral decomposition of the phase space}

In order to determine the asymptotic behavior of the semigroup $(\mathcal{U}(t))_{t \geq 0}$, we need to introduce some preliminary results. In the beginning, we introduce a definition.

Definition 8 [15, Definition 3.1, pp. 275] The operator $\mathcal{D}$ is said to be stable if there exist positive constants $\eta$ and $\mu$ such that the solution of the homogenous difference equation

$$
\left\{\begin{array}{l}
\mathcal{D} u_{t}=0, t \geq 0, \\
u_{0}=\varphi
\end{array}\right.
$$

where $\varphi \in\{\psi \in C: \mathcal{D} \psi=0\}$, satisfies

$$
\left|u_{t}(., \varphi)\right| \leq \mu e^{-\eta t}|\varphi|, \text { for } t \geq 0 .
$$

Example 9 The operator $\mathcal{D}$ defined by

$$
\mathcal{D} \varphi=\varphi(0)-q \varphi(-r)
$$

is stable if and only if $|q|<1$.

In the following, we add two supplementary assumptions

$\left(\mathbf{H}_{\mathbf{2}}\right)$ The semigroup $\left(T_{0}(t)\right)_{t \geq 0}$ is compact on $\overline{D(A)}$, for each $t>0$.

$\left(\mathbf{H}_{3}\right)$ The operator $\mathcal{D}$ is stable.

Then, we have the following fundamental result on the semigroup $(\mathcal{U}(t))_{t \geq 0}$.

Theorem 10 [6, lemma 10] Assume that $\left(\mathbf{H}_{\mathbf{0}}\right),\left(\mathbf{H}_{\mathbf{2}}\right)$ and $\left(\mathbf{H}_{\mathbf{3}}\right)$ hold. Then the semigroup $(\mathcal{U}(t))_{t \geq 0}$ is decomposed on $C_{0}$ as follows

$$
\mathcal{U}(t)=\mathcal{U}_{1}(t)+\mathcal{U}_{2}(t), \text { for } t \geq 0,
$$


where $\left(\mathcal{U}_{1}(t)\right)_{t \geq 0}$ is an exponentially stable semigroup on $C_{0}$, which means that there are positive constants $\alpha_{0}$ and $N_{0}$ such that

$$
\left|\mathcal{U}_{1}(t) \varphi\right| \leq N_{0} e^{-\alpha_{0} t}|\varphi|, \text { for } t \geq 0 \text { and } \varphi \in C_{0}
$$

and $\mathcal{U}_{2}(t)$ is compact for every $t>0$. More exactly, $\left(\mathcal{U}_{1}(t)\right)_{t \geq 0}$ is the semigroup associated to the equation

$$
\left\{\begin{array}{l}
\frac{d}{d t} \mathcal{D} u_{t}=(A+\delta I) \mathcal{D} u_{t}, t \geq 0 \\
u_{0}=\varphi
\end{array}\right.
$$

where $\delta$ is taken such that

$$
\left|e^{\delta t} T_{0}(t)\right| \leq \gamma e^{-\beta t}, \quad t \geq 0
$$

for some positive constants $\beta$ and $\gamma$.

Our next goal is to reduce Equation (1) to a finite dimensional space. We introduce the Kuratowski's measure of noncompactness $\alpha($.$) of bounded sets$ $K$ in a Banach space $Y$ by

$$
\alpha(K)=\inf \{k>0: K \text { has a finite cover of balls of diameter }<k\} \text {. }
$$

For a bounded linear operator $B$ on $Y,|B|_{\alpha}$ is defined by

$$
|B|_{\alpha}=\inf \{c>0: \alpha(B(K)) \leq c \alpha(K) \text {, for any bounded set } K \text { of } Y\} \text {. }
$$

The essential growth bound $\omega_{\text {ess }}(\mathcal{U})$ of the semigroup $(\mathcal{U}(t))_{t \geq 0}$ is defined by

$$
\begin{aligned}
\omega_{\text {ess }}(\mathcal{U}) & =\lim _{t \rightarrow+\infty} \frac{1}{t} \log |\mathcal{U}(t)|_{\alpha}, \\
& =\inf _{t>0} \frac{1}{t} \log |\mathcal{U}(t)|_{\alpha} .
\end{aligned}
$$

By Theorem 10, we deduce that $\omega_{\text {ess }}(\mathcal{U})<0$. Consequently by $[11$, Theorem 5.3.7, pp. 333], we get the following spectral decomposition.

Theorem 11 Assume that $\left(\mathbf{H}_{\mathbf{0}}\right),\left(\mathbf{H}_{\mathbf{2}}\right)$ and $\left(\mathbf{H}_{\mathbf{3}}\right)$ hold. Then $C_{0}$ is decomposed as follows

$$
C_{0}=S \oplus V
$$

where $S$ is $\mathcal{U}$-invariant and there are positive constants $\alpha$ and $N$ such that

$$
|\mathcal{U}(t) \varphi| \leq N e^{-\alpha t}|\varphi|, \text { for } t \geq 0 \text { and } \varphi \in S
$$

$V$ is a finite dimensional space and the restriction of $\mathcal{U}$ to $V$ becomes a group. 
Let $C_{0}^{*}$ be the dual space of $C_{0}$ and $d=\operatorname{dim}(V)$. Take a basis vectors $\Phi=$ $\left\{\phi_{1}, \ldots, \phi_{d}\right\}$ of $V$. Then there exist $d$-elements $\left\{\psi_{1}, \ldots, \psi_{d}\right\}$ in $C_{0}^{*}$ such that:

$$
\left\{\begin{array}{l}
\left\langle\psi_{i}, \phi_{j}\right\rangle=\delta_{i j} \\
\left\langle\psi_{i}, \phi\right\rangle=0, \text { for all } \phi \in S \text { and } i \in\{1, \ldots, d\}
\end{array}\right.
$$

where

$$
\delta_{i j}= \begin{cases}1 & \text { if } i=j \\ 0 & \text { if } i \neq j\end{cases}
$$

and $\langle.,$.$\rangle denotes the duality pairing between C_{0}^{*}$ and $C_{0}$. Let $\Psi=\operatorname{col}\left\{\psi_{1}, \ldots, \psi_{d}\right\}$ and $\langle\Psi, \Phi\rangle$ be a $d \times d$-matrix, with $\left\langle\psi_{i}, \phi_{j}\right\rangle$ its $(i, j)$-component. Then $\langle\Psi, \Phi\rangle=$ $I_{d \times d}$. Denote by $\Pi^{s}$ and $\Pi^{v}$ the projections respectively on $S$ and $V$, and by $\mathcal{U}^{s}(t)$ and $\mathcal{U}^{v}(t)$ the restrictions of $\mathcal{U}(t)$ respectively on $S$ and $V$, which correspond to the above decomposition of the phase space $C_{0}$. Let $\varphi \in C_{0}$. Then $\varphi=\Pi^{s} \varphi+\Pi^{v} \varphi$ with $\Pi^{v} \varphi=\sum_{i=1}^{d} \alpha_{i} \phi_{i}$ and $\alpha_{i} \in \mathbb{R}$. By (6), we conclude that

$$
\alpha_{i}=\left\langle\psi_{i}, \varphi\right\rangle
$$

Hence

$$
\begin{aligned}
\Pi^{v} \varphi & =\sum_{i=1}^{d}\left\langle\psi_{i}, \varphi\right\rangle \phi_{i} \\
& =\Phi\langle\Psi, \varphi\rangle .
\end{aligned}
$$

Since $\left(\mathcal{U}^{v}(t)\right)_{t \geq 0}$ is a group on $V$, then there exists a $d \times d$-matrix $G$ such that

$$
\mathcal{U}^{v}(t) \Phi=\Phi e^{t G}, \text { for } t \in \mathbb{R} .
$$

Moreover, $\sigma(G)=\left\{\lambda \in \sigma\left(\mathcal{A}_{\mathcal{U}}\right): \operatorname{Re}(\lambda) \geq 0\right\}$.

For any $n \geq n_{0}>\widetilde{\omega}$ and $i \in\{1, \ldots, d\}$, we define the linear mapping $x_{i, n}^{*}$ by

$$
x_{i, n}^{*}(a)=\left\langle\psi_{i}, \widetilde{B}_{n}\left(X_{0} a\right)\right\rangle \text {, for } a \in X .
$$

Since $\left|\widetilde{B}_{n}\right| \leq \frac{n}{n-\widetilde{\omega}} \widetilde{M}$, for any $n \geq n_{0}$, then $x_{i, n}^{*}$ is a bounded linear operator from $X$ to $\mathbb{R}$ such that

$$
\left|x_{i, n}^{*}\right| \leq \frac{n}{n-\widetilde{\omega}} \widetilde{M}\left|\psi_{i}\right|, \text { for any } n \geq n_{0} .
$$

Define the $d$-column vector $x_{n}^{*}=\operatorname{col}\left(x_{1, n}^{*}, \ldots, x_{d, n}^{*}\right)$. Then

$$
\left\langle x_{n}^{*}, a\right\rangle=\left\langle\Psi, \widetilde{B}_{n}\left(X_{0} a\right)\right\rangle, \quad a \in X
$$


This means that

$$
\left\langle x_{n}^{*}, a\right\rangle_{i}=\left\langle\psi_{i}, \widetilde{B}_{n}\left(X_{0} a\right)\right\rangle \text { for } i=1, \ldots, d \text { and } a \in X
$$

Moreover,

$$
\sup _{n \geq n_{0}}\left|x_{n}^{*}\right| \leq 2 \widetilde{M} \sup _{1 \leq i \leq d}\left|\psi_{i}\right|<\infty
$$

Which implies that $\left(x_{n}^{*}\right)_{n \geq n_{0}}$ is a bounded sequence in $\mathcal{L}\left(X, \mathbb{R}^{d}\right)$.

Theorem 12 Assume that $\left(\mathbf{H}_{\mathbf{0}}\right),\left(\mathbf{H}_{\mathbf{1}}\right),\left(\mathbf{H}_{\mathbf{2}}\right)$ and $\left(\mathbf{H}_{\mathbf{3}}\right)$ hold. There exists $x^{*} \in \mathcal{L}\left(X, \mathbb{R}^{d}\right)$, such that $\left(x_{n}^{*}\right)_{n \geq n_{0}}$ converges weakly to $x^{*}$ in the sense that

$$
\left\langle x_{n}^{*}, x\right\rangle \underset{n \rightarrow \infty}{\rightarrow}\left\langle x^{*}, x\right\rangle, \text { for all } x \in X
$$

For the proof of Theorem 12, we need the following fundamental results.

Theorem 13 [23, pp. 776] Let $Y$ be any separable Banach space and $\left(z_{n}^{*}\right)_{n \in \mathbb{N}}$ be a bounded sequence in $Y^{*}$. Then, there exists a subsequence $\left(z_{n_{k}}^{*}\right)_{k \in \mathbb{N}}$ of $\left(z_{n}^{*}\right)_{n \in \mathbb{N}}$ which converges weakly in $Y^{*}$ in the sense that there exists $z^{*} \in Y^{*}$ such that

$$
\left\langle z_{n_{k}}^{*}, x\right\rangle \underset{n \rightarrow \infty}{\rightarrow}\left\langle z^{*}, x\right\rangle \text {, for all } x \in Y \text {. }
$$

Lemma 14 Assume that $\left(\mathbf{H}_{\mathbf{0}}\right),\left(\mathbf{H}_{\mathbf{1}}\right),\left(\mathbf{H}_{\mathbf{2}}\right)$ and $\left(\mathbf{H}_{\mathbf{3}}\right)$ hold. Let $u(., \sigma, 0, f)$ be the solution of Equation (1) with $\varphi=0$. Then

$$
\Pi^{v} u_{t}(., \sigma, 0, f)=\Phi \lim _{n \rightarrow+\infty} \int_{\sigma}^{t} e^{(t-\xi) G}\left\langle x_{n}^{*}, f(\xi)\right\rangle d \xi, t \geq \sigma
$$

\section{Proof of the lemma.}

The solution $u(., \sigma, 0, f)$ of Equation ( 1$)$ with $\varphi=0$ is given, for $t \geq \sigma$, by

$$
u_{t}(., \sigma, 0, f)=\lim _{n \rightarrow+\infty} \int_{\sigma}^{t} \mathcal{U}(t-\xi)\left(\widetilde{B}_{n}\left(X_{0} f(\xi)\right)\right) d \xi
$$

Then

Since

$$
\Pi^{v} u_{t}(., \sigma, 0, f)=\lim _{n \rightarrow+\infty} \int_{\sigma}^{t} \mathcal{U}^{v}(t-\xi) \Pi^{v}\left(\widetilde{B}_{n}\left(X_{0} f(\xi)\right)\right) d \xi
$$

it follows that

$$
\Pi^{v}\left(\widetilde{B}_{n}\left(X_{0} f(\xi)\right)\right)=\Phi\left\langle\Psi, \widetilde{B}_{n}\left(X_{0} f(\xi)\right)\right\rangle=\Phi\left\langle x_{n}^{*}, f(\xi)\right\rangle,
$$

$$
\begin{aligned}
\Pi^{v} u_{t}(., \sigma, 0, f) & =\Phi \lim _{n \rightarrow+\infty} \int_{\sigma}^{t} e^{(t-\xi) G}\left\langle\Psi, \widetilde{B}_{n} X_{0} f(\xi)\right\rangle d \xi \\
& =\Phi \lim _{n \rightarrow+\infty} \int_{\sigma}^{t} e^{(t-\xi) G}\left\langle x_{n}^{*}, f(\xi)\right\rangle d \xi, t \geq \sigma
\end{aligned}
$$


Proof of Theorem 12. Let $Z_{0}$ be any closed separable subspace of $X$. Since $\left(x_{n}^{*}\right)_{n \geq n_{0}}$ is a bounded sequence, then by Theorem 13 we get that the sequence $\left(x_{n}^{*}\right)_{n \geq n_{0}}$ has a subsequence $\left(x_{n_{k}}^{*}\right)_{k \in \mathbb{N}}$ which converges weakly to some $x_{Z_{0}}^{*}$ in $Z_{0}$. We claim that the whole sequence $\left(x_{n}^{*}\right)_{n \geq n_{0}}$ converges weakly to $x_{Z_{0}}^{*}$ in $Z_{0}$. We proceed by contradiction and suppose that there exists a subsequence $\left(x_{n_{p}}^{*}\right)_{p \in \mathbb{N}}$ of $\left(x_{n}^{*}\right)_{n \geq n_{0}}$ which converges weakly to an element $\widetilde{x}_{Z_{0}}^{*}$ with $\widetilde{x}_{Z_{0}}^{*} \neq x_{Z_{0}}^{*}$. Let $a \in Z_{0}$. By Lemma 14, we get that

$$
\lim _{k \rightarrow+\infty} \int_{\sigma}^{t} e^{(t-\xi) G}\left\langle x_{n_{k}}^{*}, a\right\rangle d \xi=\lim _{p \rightarrow+\infty} \int_{\sigma}^{t} e^{(t-\xi) G}\left\langle x_{n_{p}}^{*}, a\right\rangle d \xi \text {, for } a \in Z_{0}
$$

This implies that

$$
\int_{\sigma}^{t} e^{(t-\xi) G}\left\langle x_{Z_{0}}^{*}, a\right\rangle d \xi=\int_{\sigma}^{t} e^{(t-\xi) G}\left\langle\widetilde{x}_{Z_{0}}^{*}, a\right\rangle d \xi, \text { for } a \in Z_{0}
$$

Consequently $x_{Z_{0}}^{*} \equiv \widetilde{x}_{Z_{0}}^{*}$, which gives a contradiction. We conclude that the sequence $\left(x_{n}^{*}\right)_{n \geq n_{0}}$ converges weakly to $x_{Z_{0}}^{*}$ in $Z_{0}$. Let $Z_{1}$ be anther closed separable subspace of $X$. By using the same argument as above, we get that $\left(x_{n}^{*}\right)_{n \geq n_{0}}$ converges weakly to $x_{Z_{1}}^{*}$ in $Z_{1}$. Since $Z_{0} \cap Z_{1}$ is a closed separable subspace of $X$, we get that $x_{Z_{1}}^{*} \equiv x_{Z_{0}}^{*}$ on $Z_{0} \cap Z_{1}$. For any $x \in X$, we define $x^{*}$ by

$$
\left\langle x^{*}, x\right\rangle=\left\langle x_{Z}^{*}, x\right\rangle
$$

where $Z$ is any closed separable subspace of $X$ such that $x \in Z$. Then $x^{*}$ is well defined on $X$ and it is a bounded linear mapping on $X$ such that

$$
\left|x^{*}\right| \leq \sup _{n \geq n_{0}}\left|x_{n}^{*}\right|<\infty
$$

and $\left(x_{n}^{*}\right)_{n \geq n_{0}}$ converges weakly to $x^{*}$ in $X$.

As an immediate consequence of the above theorem, we obtain that

Corollary 15 For any continuous function $h: \mathbb{R} \rightarrow X$, we have, for $t, \sigma \in \mathbb{R}$,

$$
\lim _{n \rightarrow+\infty} \int_{\sigma}^{t} \mathcal{U}^{v}(t-\xi) \Pi^{v}\left(\widetilde{B}_{n}\left(X_{0} h(\xi)\right)\right) d \xi=\Phi \int_{\sigma}^{t} e^{(t-\xi) G}\left\langle x^{*}, h(\xi)\right\rangle d \xi
$$

The expression in the above corollary is well defined for all $t, \sigma \in \mathbb{R}$, since $\left(\mathcal{U}^{v}(t)\right)_{t \in \mathbb{R}}$ is a group. We are now in the position to state a finite dimensional reduction of Equation (1).

Theorem 16 Assume that $\left(\mathbf{H}_{\mathbf{0}}\right),\left(\mathbf{H}_{\mathbf{1}}\right),\left(\mathbf{H}_{\mathbf{2}}\right)$ and $\left(\mathbf{H}_{\mathbf{3}}\right)$ hold. Let $u$ be an integral solution of Equation (1) on $\mathbb{R}$. Then, $z(t)=\left\langle\Psi, u_{t}\right\rangle$ is a solution of the 
ordinary differential equation

$$
\frac{d}{d t} z(t)=G z(t)+\left\langle x^{*}, f(t)\right\rangle, t \in \mathbb{R} .
$$

Conversely, if $f$ is a bounded function on $\mathbb{R}$ and $z$ is a solution of Equation (7) on $\mathbb{R}$, then the function u given by

$$
u(t)=\left[\Phi z(t)+\lim _{n \rightarrow+\infty} \int_{-\infty}^{t} \mathcal{U}^{s}(t-\xi) \Pi^{s}\left(\widetilde{B}_{n}\left(X_{0} f(\xi)\right)\right) d \xi\right](0), \text { for } t \in \mathbb{R},
$$

is an integral solution of Equation (1) on $\mathbb{R}$.

PROOF. Let $u$ be an integral solution of Equation (1) on $\mathbb{R}$. Then

$$
u_{t}=\Pi^{s} u_{t}+\Pi^{v} u_{t}, \text { for all } t \in \mathbb{R},
$$

and, for $t, \sigma \in \mathbb{R}$, one has

$$
\Pi^{v} u_{t}=\mathcal{U}^{v}(t-\sigma) \Pi^{v} u_{\sigma}+\lim _{n \rightarrow+\infty} \int_{\sigma}^{t} \mathcal{U}^{v}(t-\xi) \Pi^{v}\left(\widetilde{B}_{n}\left(X_{0} f(\xi)\right)\right) d \xi
$$

Since $\Pi^{v} u_{t}=\Phi\left\langle\Psi, u_{t}\right\rangle$ and by Corollary 15 , we get that

$$
\begin{aligned}
\Phi\left\langle\Psi, u_{t}\right\rangle= & \mathcal{U}^{v}(t-\sigma) \Phi\left\langle\Psi, u_{\sigma}\right\rangle+\Phi \int_{\sigma}^{t} e^{(t-\xi) G}\left\langle x^{*}, f(\xi)\right\rangle d \xi \\
& =\Phi e^{(t-\sigma) G}\left\langle\Psi, u_{\sigma}\right\rangle+\Phi \int_{\sigma}^{t} e^{(t-\xi) G}\left\langle x^{*}, f(\xi)\right\rangle d \xi
\end{aligned}
$$

Let $z(t)=\left\langle\Psi, u_{t}\right\rangle$. Then

$$
z(t)=e^{(t-\sigma) G} z(\sigma)+\int_{\sigma}^{t} e^{(t-\xi) G}\left\langle x^{*}, f(\xi)\right\rangle d \xi, \text { for } t, \sigma \in \mathbb{R} .
$$

Consequently, $z$ is a solution of the ordinary differential equation (7) on $\mathbb{R}$.

Conversely, assume that $f$ is bounded on $\mathbb{R}$. Then $\int_{-\infty}^{t} \mathcal{U}^{s}(t-\xi) \Pi^{s}\left(\widetilde{B}_{n}\left(X_{0} f(\xi)\right)\right) d \xi$ is well defined on $\mathbb{R}$. Let $z$ be a solution of $(7)$ on $\mathbb{R}$ and define $v$ by

$$
v(t)=\Phi z(t)+\lim _{n \rightarrow+\infty} \int_{-\infty}^{t} \mathcal{U}^{s}(t-\xi) \Pi^{s}\left(\widetilde{B}_{n}\left(X_{0} f(\xi)\right)\right) d \xi, \text { for } t \in \mathbb{R}
$$

Since

$$
z(t)=e^{(t-\sigma) G} z(\sigma)+\int_{\sigma}^{t} e^{(t-\xi) G}\left\langle x^{*}, f(\xi)\right\rangle d \xi, \text { for } t, \sigma \in \mathbb{R},
$$

using Corollary 15, the function $v_{1}$ given by

$$
v_{1}(t)=\Phi z(t), \text { for } t \in \mathbb{R}
$$


satisfies the integral equation

$v_{1}(t)=\mathcal{U}^{v}(t-\sigma) v_{1}(\sigma)+\lim _{n \rightarrow+\infty} \int_{\sigma}^{t} \mathcal{U}^{v}(t-\xi) \Pi^{v}\left(\widetilde{B}_{n}\left(X_{0} f(\xi)\right)\right) d \xi$, for $t, \sigma \in \mathbb{R}$

Moreover, the function $v_{2}$ given by

$$
v_{2}(t)=\lim _{n \rightarrow+\infty} \int_{-\infty}^{t} \mathcal{U}^{s}(t-\xi) \Pi^{s}\left(\widetilde{B}_{n}\left(X_{0} f(\xi)\right)\right) d \xi, \text { for } t \in \mathbb{R}
$$

satisfies

$$
v_{2}(t)=\mathcal{U}^{s}(t-\sigma) v_{2}(\sigma)+\lim _{n \rightarrow+\infty} \int_{\sigma}^{t} \mathcal{U}^{s}(t-\xi) \Pi^{s}\left(\widetilde{B}_{n}\left(X_{0} f(\xi)\right)\right) d \xi, \text { for } t \geq \sigma
$$

Then, for all $t \geq \sigma$, we have

$$
\begin{aligned}
\mathcal{U}(t-\sigma) v(\sigma) & =\mathcal{U}^{v}(t-\sigma) v_{1}(\sigma)+\mathcal{U}^{s}(t-\sigma) v_{2}(\sigma) \\
& =v_{1}(t)-\lim _{n \rightarrow+\infty} \int_{\sigma}^{t} \mathcal{U}^{v}(t-\xi) \Pi^{v}\left(\widetilde{B}_{n}\left(X_{0} f(\xi)\right)\right) d \xi+v_{2}(t)- \\
\lim _{n \rightarrow+\infty} \int_{\sigma}^{t} \mathcal{U}^{s}(t-\xi) \Pi^{s}\left(\widetilde{B}_{n}\left(X_{0} f(\xi)\right)\right) d \xi & \\
& =v(t)-\lim _{n \rightarrow+\infty} \int_{\sigma}^{t} \mathcal{U}(t-\xi)\left(\widetilde{B}_{n}\left(X_{0} f(\xi)\right)\right) d \xi .
\end{aligned}
$$

Therefore

$$
v(t)=\mathcal{U}(t-\sigma) v(\sigma)+\lim _{n \rightarrow+\infty} \int_{\sigma}^{t} \mathcal{U}(t-\xi)\left(\widetilde{B}_{n}\left(X_{0} f(\xi)\right)\right) d \xi, \text { for } t \geq \sigma .
$$

By Theorem 7, we obtain that the function $u$ defined by $u(t)=v(t)(0)$ is an integral solution of Equation (1) on $\mathbb{R}$.

\section{Almost periodic solutions for Equation (1)}

First of all, we recall some properties about almost periodic functions. Let $\mathcal{B C}(\mathbb{R}, X)$ be the space of all bounded continuous functions from $\mathbb{R}$ to $X$, provided with the uniform norm topology. For $g \in \mathcal{B C}(\mathbb{R}, X)$ and for every $\tau \in \mathbb{R}$, we define the function $g_{\tau}$ by

$$
g_{\tau}(s)=g(\tau+s), \text { for all } s \in \mathbb{R} \text {. }
$$


Definition 17 [13] A function $g$ is said to be almost periodic if the set

$$
\left\{g_{\tau}: \tau \in \mathbb{R}\right\}
$$

is relatively compact in $\mathcal{B C}(\mathbb{R}, X)$.

Consider the ordinary differential Equation (3), where $B$ is a constant $n \times n$ matrix and $g: \mathbb{R} \rightarrow \mathbb{R}^{n}$ is a continuous function.

Theorem 18 [13, Theorem 5.8, pp. 86] Assume that $g$ is an almost periodic function. Then the following are equivalent:

(i) existence of a bounded solution on $\mathbb{R}^{+}$of Equation (3),

(ii) existence of an almost periodic solution of Equation (3).

Moreover, every bounded solution on $\mathbb{R}$ is almost periodic.

For the existence of almost periodic solutions of Equation (1), we assume that

$\left(\mathbf{H}_{\mathbf{4}}\right) f$ is an almost periodic function.

Theorem 19 Assume that $\left(\mathbf{H}_{\mathbf{0}}\right),\left(\mathbf{H}_{\mathbf{1}}\right),\left(\mathbf{H}_{\mathbf{2}}\right),\left(\mathbf{H}_{\mathbf{3}}\right)$ and $\left(\mathbf{H}_{\mathbf{4}}\right)$ hold. Then the following are equivalent:

(i) existence of a bounded solution on $\mathbb{R}^{+}$of Equation (1),

(ii) existence of an almost periodic solution of Equation (1).

PROOF. Let $u$ be a bounded solution of Equation (1) on $\mathbb{R}^{+}$. By Theorem 16 , the function $z(t)=\left\langle\Psi, u_{t}\right\rangle$, for $t \geq 0$, is a solution of the ordinary differential Equation (7) and it is bounded on $\mathbb{R}^{+}$. Moreover, the function

$$
\nu(t)=\left\langle x^{*}, f(t)\right\rangle, \text { for } t \in \mathbb{R},
$$

is almost periodic from $\mathbb{R}$ to $\mathbb{R}^{d}$. By Theorem 18 , we get that the reduced system $(7)$ has an almost periodic solution $\widetilde{z}$. Consequently, $\Phi \widetilde{z}($.$) is an almost$ periodic function on $\mathbb{R}$. By Theorem 16, the function $u(t)=v(t)(0)$, where

$$
v(t)=\Phi \widetilde{z}(t)+\lim _{n \rightarrow+\infty} \int_{-\infty}^{t} \mathcal{U}^{s}(t-\xi) \Pi^{s}\left(\widetilde{B}_{n}\left(X_{0} f(\xi)\right)\right) d \xi, \text { for } t \in \mathbb{R}
$$

is an integral solution of Equation (1) on $\mathbb{R}$. To end the proof, we will show that the function

$$
y(t)=\lim _{n \rightarrow+\infty} \int_{-\infty}^{t} \mathcal{U}^{s}(t-\xi) \Pi^{s}\left(\widetilde{B}_{n}\left(X_{0} f(\xi)\right)\right) d \xi, \text { for } t \in \mathbb{R},
$$

is almost periodic. In fact for any sequence of real numbers $\left(\alpha_{p}^{\prime}\right)_{p \geq 0}$ there exists a subsequence $\left(\alpha_{p}\right)_{p \geq 0} \subset\left(\alpha_{p}^{\prime}\right)_{p \geq 0}$ such that $f\left(.+\alpha_{p}\right)$ converges uniformly on 
$\mathbb{R}$ to a function $\tilde{f}$. We can see also that $y\left(.+\alpha_{p}\right)$ converges uniformly on $\mathbb{R}$ to the function

$$
\widetilde{y}(t)=\lim _{n \rightarrow+\infty} \int_{-\infty}^{t} \mathcal{U}^{s}(t-\xi) \Pi^{s}\left(\widetilde{B}_{n}\left(X_{0} \widetilde{f}(\xi)\right)\right) d \xi, \text { for } t \in \mathbb{R} .
$$

Consequently, $y$ is an almost periodic function and $v$ is an almost periodic solution of Equation (1).

\section{Application}

In order to apply the abstract result of the previous section, we consider the model proposed in [26]:

$$
\left\{\begin{array}{l}
\frac{\partial}{\partial t}[u(t, x)-q u(t-r, x)]=\frac{\partial^{2}}{\partial x^{2}}[u(t, x)-q u(t-r, x)]+ \\
\qquad \int_{-r}^{0} G(\theta) u(t+\theta, x) d \theta+h(t, x), \text { for } t \geq \sigma \text { and } x \in[0, \pi], \\
u(t, x)-q u(t-r, x)=0, \text { for } x=0, \pi \text { and } t \geq \sigma, \\
u(\sigma+\theta, x)=\psi(\theta, x), \text { for } \theta \in[-r, 0] \text { and } x \in[0, \pi]
\end{array}\right.
$$

where $G:[-r, 0] \rightarrow \mathbb{R}, \psi:[-r, 0] \times[0, \pi] \rightarrow \mathbb{R}$ and $h: \mathbb{R} \times[0, \pi] \rightarrow \mathbb{R}$ are continuous functions $q$ is a positive constant in $(0,1)$.

In order to write System $(8)$ in an abstract form, we introduce $X=C([0, \pi] ; \mathbb{R})$ the space of continuous functions from $[0, \pi]$ to $\mathbb{R}$ endowed with the uniform norm topology. Define the operator $A: D(A) \subset X \rightarrow X$ by

$$
\left\{\begin{array}{l}
D(A)=\left\{y \in C^{2}([0, \pi] ; \mathbb{R}): y(0)=y(\pi)=0\right\} \\
A y=y^{\prime \prime}
\end{array}\right.
$$

Lemma 20 [10, Proposition 14.6] The operator A satisfies the Hille-Yosida condition on $X$ :

$$
(0,+\infty) \subset \rho(A) \text { and }\left|(\lambda I-A)^{-1}\right| \leq \frac{1}{\lambda}, \text { for } \lambda>0 .
$$

This lemma implies that condition $\left(\mathbf{H}_{0}\right)$ is satisfied. On the other hand, we 
can see that

$$
\overline{D(A)}=\{y \in X: y(0)=y(\pi)=0\} .
$$

Let us introduce the bounded linear operator $\mathcal{D}: C:=C([-r, 0] ; X) \rightarrow X$ by

$$
\mathcal{D} \phi:=\phi(0)-q \phi(-r) .
$$

Since $0<q<1$, then $\mathcal{D}$ is stable and Condition $\left(\mathbf{H}_{3}\right)$ holds. Moreover, by definitions of the operators $A$ and $\mathcal{D}$, it follows that Condition $\left(\mathbf{H}_{1}\right)$ is satisfied.

Let $L: C \rightarrow X$ be the operator defined by

$$
L(\phi)(x)=\int_{-r}^{0} G(\theta) \phi(\theta)(x) d \theta, \text { for } x \in[0, \pi] \text { and } \phi \in C,
$$

$f: \mathbb{R} \longrightarrow X$ be the mapping defined by

$$
f(t)(x)=h(t, x), \text { for } t \in \mathbb{R} \text { and } x \in[0, \pi],
$$

and the initial data $\varphi \in C$ is given by

$$
\varphi(\theta)(x)=\psi(\theta, x) \text {, for } \theta \in[-r, 0] \text { and } x \in[0, \pi] .
$$

$L$ is a bounded linear operator from $C$ to $X$. By continuity of $h$, the function $f$ is continuous from $\mathbb{R}$ to $X$. Let $w(t)=u(t,$.$) , for t \geq \sigma$. Then Equation (8) takes the abstract form

$$
\left\{\begin{array}{l}
\frac{d}{d t} \mathcal{D} w_{t}=A \mathcal{D} w_{t}+L\left(w_{t}\right)+f(t), \text { for } t \geq \sigma \\
w_{\sigma}=\varphi \in C
\end{array}\right.
$$

Let $A_{0}$ be the part of the operator $A$ in $\overline{D(A)}$. Then $A_{0}$ is given by

$$
\left\{\begin{array}{l}
D\left(A_{0}\right)=\left\{y \in C^{2}([0, \pi] ; \mathbb{R}): y(0)=y(\pi)=y^{\prime \prime}(0)=y^{\prime \prime}(\pi)=0\right\}, \\
A_{0} y=y^{\prime \prime}, \text { for } y \in D\left(A_{0}\right) .
\end{array}\right.
$$

It is well known from [11, Example 1.4.34, pp. 123], that the operator $A_{0}$ generates a strongly continuous compact semigroup $\left(T_{0}(t)\right)_{t \geq 0}$ on $\overline{D(A)}$ and

$$
\left|T_{0}(t)\right| \leq e^{-t}, \text { for } t \geq 0 .
$$

This implies that $\left(\mathbf{H}_{\mathbf{2}}\right)$ holds. By Theorem 3, for any $\varphi \in C$ such that

$$
\mathcal{D} \varphi \in\{y \in X: y(0)=y(\pi)=0\}
$$

there exists a unique integral solution of Equation (9) on $[-r+\sigma,+\infty)$.

In order to study the existence of almost periodic solutions of Equation (9), 
we suppose that

$\left(\mathbf{H}_{\mathbf{5}}\right) h$ is almost periodic in $t$ uniformly for $x \in[0, \pi]$, which means that for any $\varepsilon>0$, there is a positive number $l(\varepsilon)$, such that any interval of length $l(\varepsilon)$ contains a $\tau$ for which

$$
|h(t+\tau, x)-h(t, x)|<\varepsilon, \text { for all }(t, x) \in \mathbb{R} \times[0, \pi] .
$$

By Assumption $\left(\mathbf{H}_{\mathbf{5}}\right)$, we deduce that the function $f: \mathbb{R} \rightarrow X$ is almost periodic.

Moreover, we suppose that

$\left(\mathbf{H}_{\mathbf{6}}\right)$ there exists a constant $\beta \in(0,1)$ such that

$$
\int_{-r}^{0}|G(\theta)| d \theta \leq(1-q) \beta
$$

Proposition 21 Assume that $\left(\mathbf{H}_{\mathbf{5}}\right)$ and $\left(\mathbf{H}_{\mathbf{6}}\right)$ hold. Then, Equation (9) has a bounded solution on $\mathbb{R}^{+}$, and it has an almost periodic solution.

Proof. The first step is to prove that Equation (9) has a bounded solution on $\mathbb{R}^{+}$. Let

$$
\rho=\frac{1}{1+q}\left(1+\frac{|f|}{1-\beta}\right),
$$

where $|f|=\sup _{s \in \mathbb{R}}|f(s)|$. Take $\varphi \in C$ such that $|\varphi|<\rho$. Then

$$
|\varphi(0)-q \varphi(-r)|<(1+q) \rho
$$

Let $w$ be the integral solution of Equation (9) with the initial condition $\varphi$. We claim that

$$
|w(t)-q w(t-r)| \leq(1+q) \rho, \text { for all } t \geq 0 .
$$

We proceed by contradiction. Let $t_{0}$ be the first time such that (11) is not true. Then

$$
t_{0}=\inf \{t>0:|w(t)-q w(t-r)|>(1+q) \rho\}
$$

By continuity, one has

$$
\left|w\left(t_{0}\right)-q w\left(t_{0}-r\right)\right|=(1+q) \rho
$$

and there exists a positive constant $\varepsilon>0$ such that

$$
|w(t)-q w(t-r)|>(1+q) \rho, \text { for } t \in\left(t_{0}, t_{0}+\varepsilon\right) .
$$


Using the variation of constants formula, we obtain $\left|w\left(t_{0}\right)-q w\left(t_{0}-r\right)\right| \leq e^{-t_{0}}(1+q) \rho+\int_{0}^{t_{0}} e^{-\left(t_{0}-s\right)}\left[\int_{-r}^{0}|G(\theta)||u(s+\theta)| d \theta+|f|\right] d s$.

Since $|w(t)-q w(t-r)| \leq(1+q) \rho$, for $t \leq t_{0}$, then

$$
|w(t)| \leq(1+q) \rho+q|w(t-r)|, \text { for } t \in\left[-r, t_{0}\right] .
$$

Moreover, since $|\varphi|<\rho$, we can see that

$$
|w(t)| \leq \frac{1+q}{1-q} \rho, \text { for } t \in\left[-r, t_{0}\right]
$$

Then

$$
\left|w\left(t_{0}\right)-q w\left(t_{0}-r\right)\right| \leq e^{-t_{0}}(1+q) \rho+\left(1-e^{-t_{0}}\right)\left[\int_{-r}^{0}|G(\theta)| d \theta \frac{1+q}{1-q} \rho+|f|\right] .
$$

Condition $\left(\mathbf{H}_{\mathbf{6}}\right)$ implies that

$$
\left|w\left(t_{0}\right)-q w\left(t_{0}-r\right)\right| \leq e^{-t_{0}}(1+q) \rho+\left(1-e^{-t_{0}}\right)((1+q) \beta \rho+|f|) .
$$

Thanks to (10), we obtain

$$
\left|w\left(t_{0}\right)-q w\left(t_{0}-r\right)\right| \leq e^{-t_{0}}(1+q) \rho+\left(1-e^{-t_{0}}\right)(1+q) \rho-\left(1-e^{-t_{0}}\right)(1-\beta) .
$$

Consequently, we obtain that

$$
\left|w\left(t_{0}\right)-q w\left(t_{0}-r\right)\right| \leq(1+q) \rho-\left(1-e^{-t_{0}}\right)(1-\beta)<(1+q) \rho .
$$

By continuity, there exists a positive $\varepsilon_{0}$ such that

$$
|w(t)-q w(t-r)|<(1+q) \rho, \text { for } t \in\left(t_{0}, t_{0}+\varepsilon_{0}\right) .
$$

Which gives a contradiction. We deduce that

$$
|w(t)-q w(t-r)| \leq(1+q) \rho, \text { for } t \geq 0 .
$$

We claim that

$$
|w(t)| \leq \frac{1+q}{1-q} \rho, \text { for } t \geq 0
$$

Let $t \in[0, r]$. Then

$$
|w(t)| \leq(1+q) \rho+q \rho \leq(1+q)(1+q) \rho,
$$

and for $t \in[r, 2 r]$

$$
|w(t)| \leq(1+q)\left(1+q+q^{2}\right) \rho .
$$

We proceed by steps and we obtain that for $t \in[(n-1) r, n r]$ 


$$
|w(t)| \leq(1+q)\left(1+q+q^{2}+\ldots+q^{n}\right) \rho .
$$

Consequently,

$$
|w(t)| \leq(1+q) \rho \sum_{n \geq 0} q^{n}=\frac{1+q}{1-q} \rho, \text { for all } t \geq 0 .
$$

Then Equation (9) has a bounded integral solution $w$ on $\mathbb{R}^{+}$. By Theorem 19, we deduce that Equation (9) has an almost periodic solution.

\section{References}

[1] M Adimy and K. Ezzinbi, Equation de type neutre et semi groupes intégrés, C.R. Acad. Sci. Paris, t. 318, Série I, 529-534, (1994).

[2] M. Adimy and K. Ezzinbi, A class of linear partial neutral functional differential equations with non-dense domain, Journal of Differential Equations, Vol. 147, $\mathrm{N}^{0} .2,285-332,(1998)$.

[3] M Adimy and K. Ezzinbi, Strict solutions of nonlinear hyperbolic neutral differential equations, Applied Mathematics Letters, 12, 107-112, (1999).

[4] M. Adimy and K. Ezzinbi, Existence and linearized stability for partial neutral functional differential equations, Differential Equations and Dynamical Systems, Vol. 7, 371-417, (1999).

[5] M. Adimy and K. Ezzinbi, Existence and stability of solutions for a class of partial neutral functional differential equations, Hiroshima Mathematical Journal, (to appear 2004).

[6] M. Adimy, K. Ezzinbi and M. Laklach, Spectral decomposition for partial neutral functional differential equations, Canadian Applied Mathematics Quarterly, Vol. $9, \mathrm{~N}^{0} .1,1-34$, Spring (2001).

[7] W. Arendt, C.J.K. Batty, M. Hieber and F. Neubrander, Vector-Valued Laplace Transforms and Cauchy Problems, Monographs in Mathematics, Vol. 96, Birkhäuser Verlag, (2001).

[8] R. Benkhalti and K. Ezzinbi, A Massera type criterion for some partial functional differential equations, Dynamic Systems and Application, Vol. 9, N0 . 2, 221-228, (2000).

[9] R. Benkhalti, H. Bouzahir and K. Ezzinbi, Existence of a periodic solution for some partial functional differential equations with infinite delay, Journal of Mathematical Analysis and Applications, Vol. 256, N0.1, 257-280, (2001).

[10] G. Da Prato and E. Sinestrari, Differential operators with non dense domain, Annali Scuola Normale Superiore di Pisa, Vol. 14, N0. 2, 285-344, (1987). 
[11] K.-J. Engel, R. Nagel, One-Parameter Semigroups of Linear Evolution Equations, Graduate Texts in Mathematics, Vol. 194, Springer-Verlag, (2000).

[12] K. Ezzinbi and J. H. Liu, Periodic solutions of non-densely defined delay evolution equations, Journal of applied Mathematics and Stochastic Analysis, Vol. 15, N $.2,113-123,(2002)$.

[13] A. Fink, Almost Periodic Differential Equations, Lectures Notes, Vol. 377, Springer-Verlag (1974).

[14] T. Furumouchi, T. Naito, N. V. Minh, Boundedness and almost periodicity of solutions of partial functional differential equations, Journal of Differential Equations, Vol. 180, N0. 1, 125-152, (2002).

[15] J. K. Hale and S. M. Verduyn-Lunel, Introduction to Functional Differential Equations, Applied Mathematical Sciences, Vol. 99, Springer-Verlag, (1993).

[16] J. K. Hale, Partial neutral functional differential equations, Rev. Roumaine Math. Pure Appli., Vol. 39, N. 4, 339-344, (1994).

[17] J. K. Hale, Coupled oscillators on a circle, Resenhas Inst. Mat. Estat. Univ. São Paulo, Vol. 1, Nº. 4, 441-457, (1994).

[18] Y. Hino and S. Murakami, Existence of an almost periodic solution of abstract linear functional differential equations with infinite delay, Dynamic Systems and Applications, Vol. 5, $\mathrm{N}^{0} .3,385-399$, (1996).

[19] V. Lakshmikantham and S. Leela, Almost periodic systems and differential inequalities, J. Math. Phys. Sci. 1, 186-301, (1967).

[20] J. L. Massera, The existence of periodic solutions of systems of differential equations, Duke Math., J. 17, 457-475, (1950).

[21] S. Murakami, T. Naito, N. V. Minh, Massera Theorem for almost periodic solutions of functional differential equations, Journal of Mathematical Society of Japan, Vol. 56, 247-268, (2004).

[22] C. C. Travis and G. F. Webb, Existence and stability for partial functional differential equations, Transactions of the American Mathematical Society, Vol. 200, 395-418, (1974).

[23] E. Zeidler, Nonlinear Functional Analysis and its Applications, Tome I, Fixed Point Theorems, Springer-Verlag, (1993).

[24] G. F. Webb, Asymptotic stability for abstract nonlinear functional differential equations, Proceeding of the American Mathematical Society, Vol. 54, 225-230, (1976).

[25] J. Wu, Theory and Applications of Partial Functional Differential Equations, Vol. 119, Springer-Verlag, (1996).

[26] J. Wu and H. Xia, Self-sustained oscillations in a ring array of coupled lossless transmission lines, Journal of Differential Equations, Vol. 124, N0. 1, 247-278, (1996). 
[27] J. Wu and H. Xia, Rotating waves in neutral partial functional differential equations, Journal of Dynamics and Differential Equations, Vol. 11, $\mathrm{N}^{0}$. 2, 209238, (1999). 